\title{
A stringent upper limit of 20 pptv for methane on Mars and constraints on its dispersion outside Gale crater
}

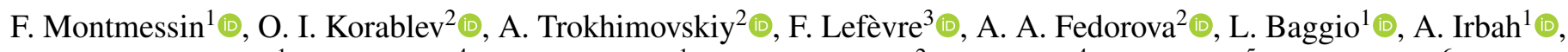

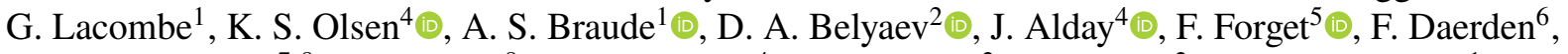 \\ J. Pla-Garcia ${ }^{7,8}$ @ , S. Rafkin ${ }^{8} \odot$, C. F. Wilson ${ }^{4}$, A. Patrakeev² , A. Shakun ${ }^{2}$, and J. L. Bertaux ${ }^{1}$ \\ ${ }^{1}$ LATMOS/IPSL, UVSQ Université Paris-Saclay, Sorbonne Université, CNRS, Guyancourt, France \\ e-mail: franck.montmessin@latmos.ipsl.fr \\ 2 Space Research Institute (IKI) RAS, Moscow, Russia \\ ${ }^{3}$ LATMOS/IPSL, Sorbonne Université, UVSQ Université Paris-Saclay, CNRS, Paris, France \\ ${ }^{4}$ AOPP, Oxford University, Oxford, UK \\ ${ }^{5}$ Laboratoire de Météorologie Dynamique, Sorbonne Université, Paris, France \\ ${ }^{6}$ BIRA-IASB, Bruxelles, Belgium \\ ${ }^{7}$ Centro de Astrobiología (CSIC?INTA), Torrejón de Ardoz, Spain \\ ${ }^{8}$ Southwest Research Institute, Boulder, CO, USA
}

Received 20 January 2021 / Accepted 23 April 2021

\begin{abstract}
Context. Reports on the detection of methane in the Martian atmosphere have motivated numerous studies aiming to confirm or explain its presence on a planet where it might imply a biogenic or more likely a geophysical origin.

Aims. Our intent is to complement and improve on the previously reported detection attempts by the Atmospheric Chemistry Suite (ACS) on board the ExoMars Trace Gas Orbiter (TGO). This latter study reported the results of a campaign that was a few months in length, and was significantly hindered by a dusty period that impaired detection performances.

Methods. We unveil 640 solar occultation measurements gathering 1.44 Martian years worth of data produced by the ACS.

Results. No methane was detected. Probing the clear northern summer season allowed us to reach $1 \sigma$ upper limits of around $10 \mathrm{pptv}$ $(20 \mathrm{pptv}$ at $2 \sigma$ ), with an annual mean of the smallest upper limits of $20 \mathrm{pptv}$. Upper limits are controlled by the amount of dust in the atmosphere, which impairs detection performance around the equator and during the southern spring and summer seasons. Observations performed near Gale crater yielded $1 \sigma$ upper limits of up to four times less than the background values measured by the Curiosity rover during the corresponding seasons.

Conclusions. Reconciliation of the absence of methane in the TGO spectra with the positive detections by Curiosity is even more difficult in light of this annual survey performed by ACS. Stronger constraints are placed on the physical and chemical mechanism capable of explaining why the mean of the best overall upper limits of ACS is ten times below the smallest methane abundances measured by Curiosity.
\end{abstract}

Key words. planets and satellites: atmospheres - planets and satellites: terrestrial planets

\section{Introduction}

The search for methane on Mars has followed a long and tortuous path towards what could potentially be considered a major discovery for Mars' exploration. The first attempt at finding methane dates back to the Mariner 7 mission (Sullivan 1969). Two days after the Mariner 7 flyby of Mars, a public announcement of methane detection was released before the Mariner 7 team realised that the infrared spectral signature attributed to atmospheric methane was, in reality, produced by $\mathrm{CO}_{2}$ ice (Herr and Pimentel, 1969). Since then, many teams have searched for methane using ground-based observatories as well as orbital and landed assets, basing their analysis on mid- and thermal-infrared wavelengths where methane possesses strong and distinctive absorption signatures. An annotated list of these attempts can be found in Zahnle et al. (2011), Knutsen et al. (2021), and in tabulated form in Pla-Garcia et al. (2019).

Hitchcock \& Lovelock (1967) pointed out that the composition of the Martian atmosphere could provide clues as to the possible presence of life on the planet. Therefore, methane has long been seen as the most accessible testimony of potential signs of life (Atreya et al. 2003, 2007) or of a remnant geophysical activity on Mars. Indeed, the presence of methane has such a strong connotation of Mars being potentially an active or even a life-hosting planet, contrary to all other chemical or geophysical evidence suggesting the opposite, that it has attracted the attention of a wide community extending far beyond those interested in Martian atmospheric composition.

However, the succession of failed and successful attempts to detect methane has also created a blurred picture, and a comprehensive understanding of the existence and origin of methane on Mars has remained out of reach. Several attempts that were initially presented as positive detections were later either retracted or questioned. This is the case of the Mariner 7 detection (as explained above) and the Canadian-France-Hawaii Telescope (CFHT) observations of Krasnopolsky et al. (2004) - whose spectrum quality raised the suspicion of Zahnle et al. (2011), who also questioned the seasonal variations of methane found by Geminale et al. (2008) based on a survey carried out with the Planetary Fourier Spectrometer (PFS). Finally, the methane 
mapping of Fonti \& Marzo (2010) using the Thermal Emission Spectrometer (TES) was later retracted by the same authorsin Fonti et al. (2015). Of all the detections made between 2003 and 2010 , only a few survived the scrutiny of the community. The PFS 2003 detection reported in Formisano et al. (2004), while bearing evident shortcomings regarding its resolving power and sensitivity, has resisted criticism and remains to this date the first non-rebutted detection of methane in the Martian atmosphere. In addition, Mumma et al. (2009) published the first ground-based evidence of methane collected the same year as the PFS, the scientific implications of which were bolstered by the revelation of puzzling spatial and seasonal variations attributed to a sudden release from the Nili Fossae region.

Several studies have explored the chemical ramifications (Lefèvre \& Forget 2009) as well as the spectroscopic consistency of these detections (Zahnle et al. 2011). These works converged toward the idea that our current understanding of the physics and chemistry governing the Martian atmosphere is incompatible with the observed variable behaviour of methane, which should rely on an extraordinary, yet undefined mechanism. Together with the fact that detections had long lain on the fringe of the detection capability of all instruments used at that time, the methane story has slowly and relentlessly created a schism in the Martian community between those convinced of its existence and those asking for more evidence. This lack of consensus was the basic justification for sending an orbiter to Mars with the sole intent of establishing the most accurate detection and the most stringent upper limits of an exhaustive list of trace gases with demonstrated relations to life or to (sub)surface activity, including methane, organic, sulphuric, and halogen molecules (Zurek et al. 2011). Launched in March 2016, the TGO mission of the joint European Space Agency (ESA) and Roscosmos ExoMars endeavour to Mars was designed to primarily serve this very objective as well as to serve as a telemetry relay to landed missions.

Meanwhile, the Mars Science Laboratory (MSL) mission with its rover Curiosity had already landed on Mars and had tested the existence of methane for the first time at the surface of Mars with the Tunable Laser Spectrometer (TLS), a part of the Sample Analysis at Mars (SAM) instrument. The TLS delivered a series of results, starting with non-detections (Webster et al. 2013), that later turned into a couple of methane spikes (Webster et al. 2015) and were finally complemented by higher sensitivity measurements revealing a seasonally varying background whose relative abundance was one order of magnitude weaker than the spikes (Webster et al. 2018, hereafter W18). Interestingly, concurrent measurements by the PFS provided orbital confirmation of one of the 2013 spikes of methane above Gale crater (Giuranna et al. 2019). The largest methane spike recorded by the TLS of $19 \pm 0.18$ parts per billion by volume (ppbv) was reported in Moores et al. (2019), corresponding to measurements carried out on 20 June 2019.

Overall, detections reported so far show methane relative abundances ranging from 0.2 (W18) to 45 ppbv (Mumma et al. 2009) and are characterised by their strong temporal and spatial variability. For Gale in particular, the picture of a crater hosting or traversed by intermittent methane releases combined with a continuous outgassing has slowly emerged.

However, no part of the global methane picture has been corroborated by the TGO. Korablev et al. (2019, hereafter K19) described the first methane campaign conducted by two independent teams using the two TGO infrared spectrometers, ACS (Atmospheric Chemistry Suite) and NOMAD (Nadir and Occultation for MArs Discoveries), between solar longitude $\left(\mathrm{L}_{\mathrm{s}}\right)$ $164^{\circ}$ and $241^{\circ}$ of Martian year (MY) 34, which is from April to October 2018. TGO measurements indicated that methane was absent above a 50 parts per trillion by volume (pptv) level on average (the minimum upper limit was found to be $12 \mathrm{pptv}$ ), which is 10 to 1000 times smaller than other reported detections. This upper limit was later corroborated by an additional study conducted with NOMAD encompassing a period comparable to that presented here, reporting a minimum upper limit of 60 pptv for methane (Knutsen et al. 2021), in line with K19. K19 stressed the difficulty in reconciling TGO upper limits with MSL detections, as these latter implied that background methane was only emitted inside or around Gale crater for no more than $25 \mathrm{yr}$ to remain under the detection level of TGO, neglecting the contribution of spikes which could only shorten this duration.

This conundrum in the search for methane on Mars remains incompletely solved because of the strong assumptions that the proposed explanations rely on (W18; Moores et al. 2019). In particular, the fact that Gale could be the only place on Mars where methane is released is a hypothesis that is difficult, if not impossible, to justify. In addition, the simultaneous existence of spikes and a background of methane is ruled out by the atmospheric circulation configuration predicted for Gale (Pla-Garcia et al. 2019).

The purpose of the present study is to follow up on the K19 study with ACS methane detection attempts aggregated over 16 Martian months. Relying on solar occultation spectra collected in the mid-infrared range bracketing the $v_{3}$ asymmetric stretching vibration band of methane centred around $3000 \mathrm{~cm}^{-1}$ $(3.3 \mu \mathrm{m})$, we describe a dataset encompassing the April 2018 to November 2020 period, completing the dataset used in K19 that only covered a quarter of MY34, essentially probing the dusty conditions of the 2018 global dust storm (Guzewich et al. 2019).

The first part of the present article concerns the technical description of ACS and of the methodology employed to convert the ACS methane detection attempts into potential detections or upper limits. The results derived from this dataset are then described in terms of its temporal and spatial dimensions with a focus on a series of measurements performed around the Gale crater. The implications of the results obtained by ACS in its search for methane for more than one Mars year are then presented and compared to other measurements, in particular those of MSL, and to the previous results described in K19. A summary of our findings is then given in Sect. 5.

\section{ACS observations}

\subsection{Dataset}

The dataset used for our analysis comprises 640 Solar occultation sequences performed by ACS between 22/04/2018 and $27 / 11 / 2020$, For these observations, ACS was set to observe the 2900-3200 $\mathrm{cm}^{-1}$ wavenumber region encompassing the entire $v_{3}$ asymmetric stretching vibration band of methane (see Fig. 1).

ACS is a cross-dispersion grating spectrometer which couples two gratings dispersing light in orthogonal directions to spatially separate the various diffraction orders of the main echelle grating and give access to a large spectral range (a multiple of the free spectral range of the echelle grating) with fine spectral sampling. This creates a 2D spectrum stacking spectral segments (or diffraction orders) along the $y$-axis, each segment being dispersed along the $x$-axis (Korablev et al. 2018). These images are displayed over a $640 \times 512 \mathrm{HgCdTe}$ pixel array (the Scorpio model of Lynred, formerly known as Sofradir) 


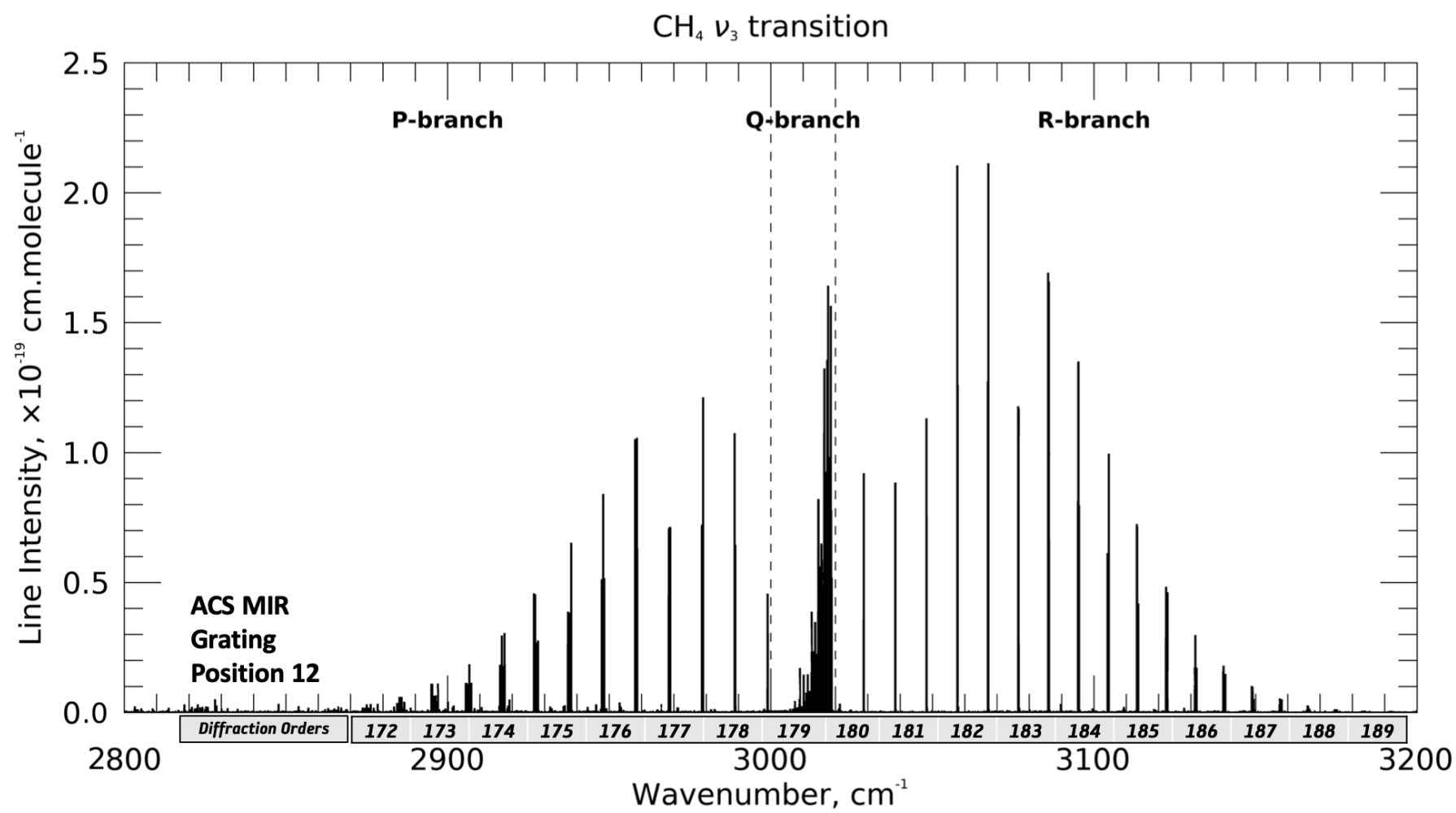

Fig. 1. Wavelength range used for the present study where the $v_{3}$ asymmetric $\mathrm{C}-\mathrm{H}$ stretching vibration band of $\mathrm{CH}_{4}$ is located. ACS spectral coverage is shown for indication with a discretization into spectral segments corresponding to the diffraction orders sampled by the grating position 12 set-up. The $Q$-branch, which comprises the densest portion of the band, is located in orders 179 and 180 whereas the most intense lines of the $v_{3}$ transition can be found in the $R$-branch and are sampled by order 182 .

with a $15 \mu \mathrm{m}$ pitch. Each diffraction order covers approximately 20 lines of detector along the slit, each line corresponding to a $0.15 \mathrm{mrad}$ aperture directed to a point on the Sun.

In position 12 of the secondary grating, which is used to observe the $v_{3}$ transition of methane, 21 diffraction orders from 172 to 192 are densely displayed, leading to a partial overlap between adjacent orders. In addition, the image around the centre of the slit, where the signal is maximum, is affected by a doubling that can be handled by a double Gaussian representation of the instrument line shape (ILS), as explained in Alday et al. (2019) and Olsen et al. (2020); Olsen et al. (2021). However, a simpler approach, though one that does not take advantage of the optimal signal-to-noise ratio $(\mathrm{S} / \mathrm{N})$ offered by the instrument, consists in selecting the two detector rows located respectively 5 and 6 rows below the row peaking in intensity, which provides the best trade-off between the doubling and overlapping phenomena. Even if the Sun signal is dimmer in that part, the ILS can be simply represented by a unique Gaussian whose full width at half maximum (FWHM) is roughly equal to $0.1 \mathrm{~cm}^{-1}$.

ACS transmission spectra are assembled by ratioing spectra observed below an altitude of $150 \mathrm{~km}$ (where atmospheric absorption is imperceptible) with those collected above, where absorption by gas and particles is negligible. However, resulting transmittances usually feature a distorted continuum appearance that needs to be corrected to mitigate biases in the retrieval. To this end, an estimate of the continuum is constructed by finding and connecting local maxima throughout the spectra and performing an 11-pixel-wide smoothing of the interpolated curve. The observed spectrum is then divided by this smoothed curve, yielding a normalised transmittance corrected for continuum distortions.
One of the last steps of the pre-processing concerns the assignment of error bars to data. The S/N obtained by the ACS is empirically estimated in any given spectrum by selecting an interval expected to be free of gaseous absorption and by estimating the statistical dispersion of the high-frequency component of the spectrum. The latter is extracted by subtracting a version of the spectrum smoothed with an 11-pixel-wide running average to the actual spectrum. However, this empirical estimation, which assumes that no correlated signal perturbation exists beyond 11 pixels, is further refined at a later stage of the processing (see Sect. 2.3). A typical $\mathrm{S} / \mathrm{N}$ altitude profile is characterised by a value of 5000 to 10000 (depending on diffraction order) in the non-absorbing portion of the occultation, and gradually reduces as the line of sight (LOS) approaches the surface, intersecting the denser and dustier layers of the atmosphere. Depending on season and location, the atmosphere can be transparent enough to allow the Sun to be observed down to the near-surface $(<5 \mathrm{~km})$. A good, though not isolated, example of these transparent windows can be found in the $60^{\circ}$ to $80^{\circ}$ northern latitudinal band near the equinox. On the other hand, dusty conditions typical of the southern spring and summer can prevent sounding of the atmosphere below $30 \mathrm{~km}$. Also, the equatorial region, even during the clearest period of the year, always exhibits significant dust and cloud opacity that degrades ACS detection performances in comparison with higher latitudes.

All the locations corresponding to the detection attempts are displayed in Fig. 2, which shows that while most of the Martian globe was sampled at some point, the intertropical region received less attention as a consequence of the TGO orbit combined with the geometrical constraints of occultations. The dataset covers 1.44 MY (2.5 Earth years) spanning from $\mathrm{L}_{\mathrm{s}}$ 


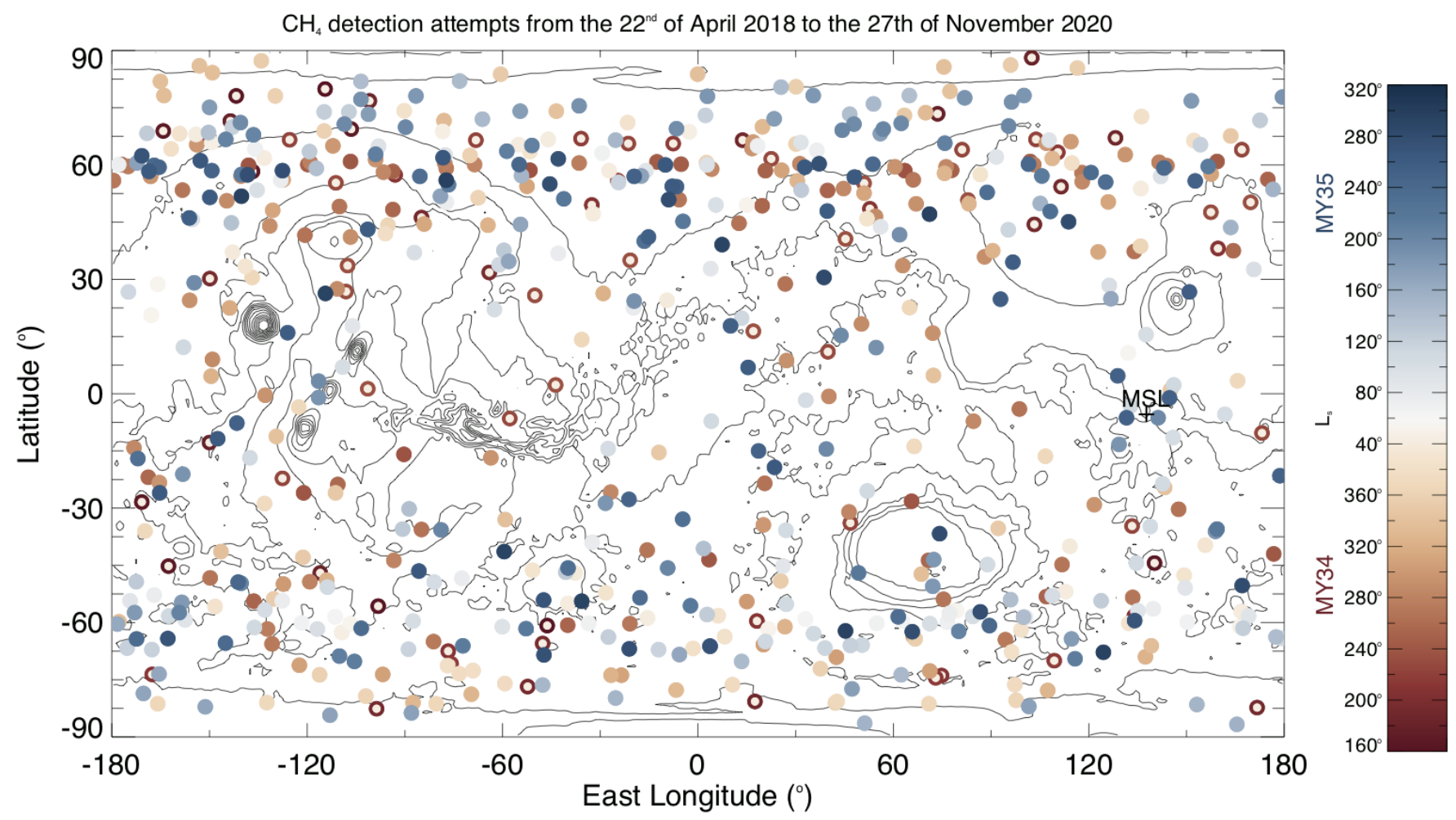

Fig. 2. Map showing the locations of all the $\mathrm{ACS} \mathrm{CH}_{4}$ detection attempts described in this article. Filled circles and ring symbols are coloured as a function of $L_{s}$. Rings correspond to measurements reported in Korablev et al. (2019).

$163.2^{\circ}$ of MY 34 to $\mathrm{L}_{\mathrm{s}} 321.9^{\circ}$ of MY 35 , which constitutes a fivefold extension to the first ACS dataset dedicated to methane and presented in K19.

\subsection{Retrieval method}

The retrieval method applied to the ACS occultation dataset is based on the one described in K19. It consists of a LevenbergMarquardt algorithm adjusting parameters to fit the observed transmittance spectrum with a model representing the instrumental convolution of a line-by-line spectroscopic model based on the HITRAN 2016 database (see two examples given in Fig. 3). The species taken into account are $\mathrm{CO}_{2}, \mathrm{H}_{2} \mathrm{O}, \mathrm{CH}_{4}$, and $\mathrm{O}_{3}$. The dependence of molecular absorption cross-sections with pressure is included by relying on the LMD Mars Climate Model predictions for MY34 and 35 (Forget et al. 1999; Montabone et al. 2020).

The retrieval process can be broken down into several steps. First, a correction is implemented for the micro spectral drift occurring during the occultation, which is due to thermomechanical displacements of the optical bench at a rate of approximately 0.01 pixel per acquisition. A precise wavelength calibration is subsequently established by fitting strong water vapour lines bracketing the interval of interest with a set of parameters representing the wavelength-to-pixel assignment as a second-order polynomial. The spectrum chosen to perform this spectral calibration is taken at the altitude where the deepest water absorption is found. The retrieved set of parameters is then assumed to remain constant with altitude and a fixed wavelength-to-pixel assignment is applied.

In order to derive a volume mixing ratio (VMR), one has to determine the corresponding $\mathrm{CO}_{2}$ slant column density. To this end, we make use of the $628 \mathrm{CO}_{2}$ isotopologue band system prevailing in order 178 that was discovered on Venus by two independent teams (Bertaux et al. 2008; Villanueva et al. 2008) and exhibits a pronounced $Q$-branch located at $2982 \mathrm{~cm}^{-1}$ (see Fig. 3)

Since K19, two studies have revealed that the methane region unexpectedly hosts absorption features of two well-known Martian compounds, $\mathrm{CO}_{2}$ and $\mathrm{O}_{3}$. Trokhimovskiy et al. (2020), using ACS data, showed that the $Q$-branch part of the methane band contains regular absorption features that were previously undocumented and that happen to correspond to the magnetic quadrupole transition of $\mathrm{CO}_{2}$ as detailed in Perevalov et al. (2021). Simultaneously, Olsen et al. (2020), also using the ACS, showed that the $Q$ - and $R$-branch ranges of methane are affected by ozone continuum absorption. These two findings, besides their implication for previous and debated methane detection attempts on Mars (Webster et al. 2020), have led to the implementation of these so far unaccounted species as additional gaseous parameters to provide a better fit.

Diffraction order $182\left(3045-3065 \mathrm{~cm}^{-1}\right)$ located in the $R$ branch domain hosts the most intense $\mathrm{CH}_{4}$ lines and is also unaffected by the recently discovered $\mathrm{CO}_{2}$ lines, hosting only $\mathrm{O}_{3}$ in addition to $\mathrm{H}_{2} \mathrm{O}$. For this reason, it was chosen as the reference order for the methane retrieval. Other orders (183, $3065-3085 \mathrm{~cm}^{-1}$, and $184,3080-3100 \mathrm{~cm}^{-1}$ ) were additionally used to provide independent confirmation were methane detected from order 182. However, these orders were not considered in our upper limit determination given their reduced sensitivity to $\mathrm{CH}_{4}$ compared to order 182 .

All retrieval attempts are performed in a LOS-integrated sense which gives access to the entire quantity of gas along the LOS per unit surface. The VMR is obtained after ratioing the derived LOS concentration of the gas with that of $\mathrm{CO}_{2}$ obtained simultaneously. It can be shown theoretically that the bias induced by this approach does not exceed 5-10\% compared to the actual or local VMR pertaining to the tangent point. In 

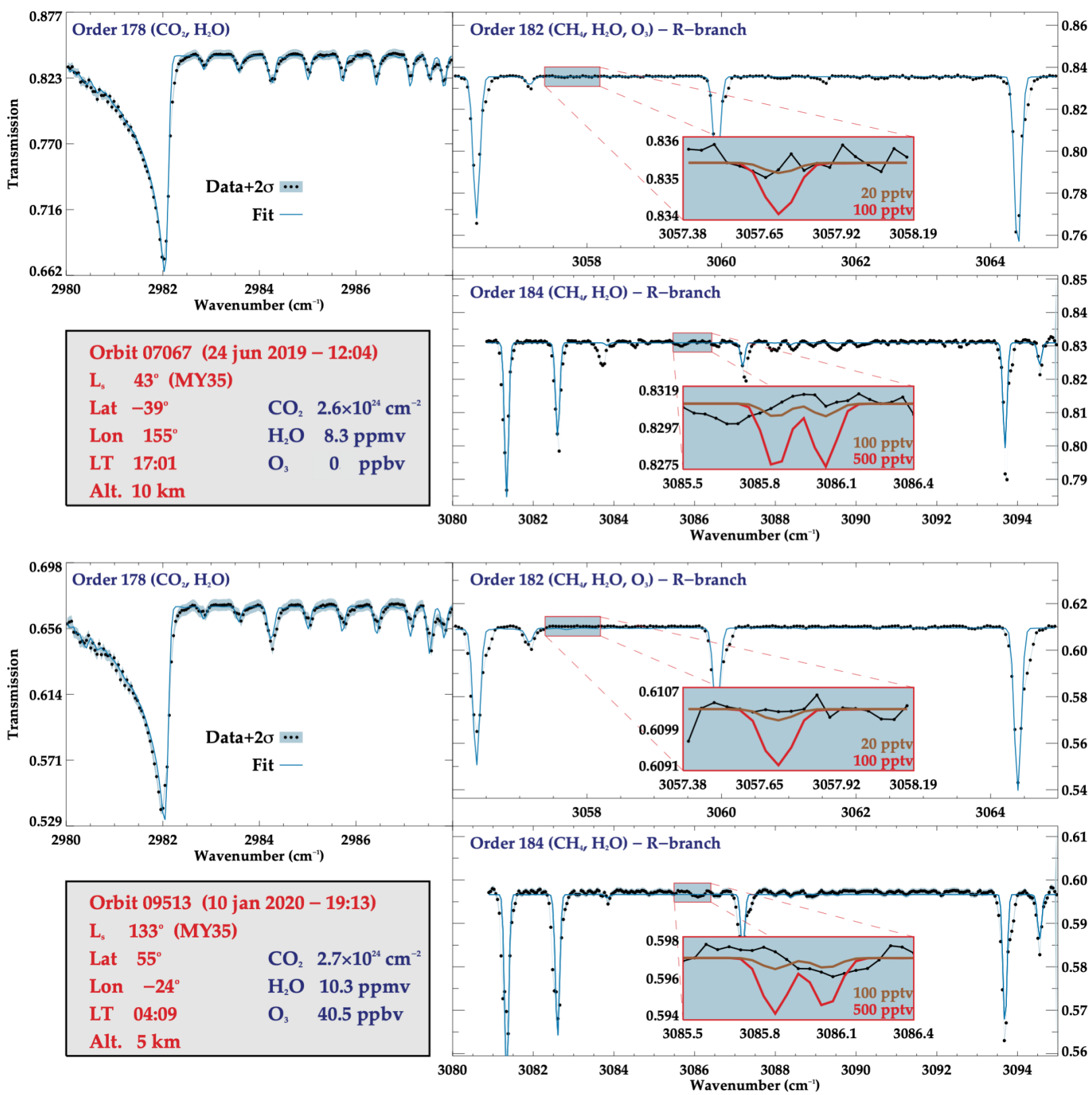

Fig. 3. ACS spectra and their associated best fits for two orbits (7067 and 9513) that occurred during the aphelion period of MY35. Diffraction orders 178, 182, and 184 are displayed for each orbit. Order 178 allows the retrieval of $\mathrm{CO}_{2}$ that is then used to compute $\mathrm{CH}_{4}$ upper limits in relative abundance. Order 182 hosts the strongest lines of the $v_{3}$ band system and yields the smallest upper limits. The noisier order 184 possesses a distinct double-lobe absorption feature that can be used to discriminate the presence of $\mathrm{CH}_{4}$ at a level that is around five times higher than in Order 182 . Notional $\mathrm{CH}_{4}$ absorptions (20 and 100 pptv for order 182, and 100 and 500 pptv for order 184) against the observed spectra and centred around the strongest feature of each order are indicated in the small insets.

addition, such an approach confers the benefit that it can be used to determine an upper limit from a single spectrum, whereas doing it in a local sense requires isolation of the concentration in the tangent layer from those of the other layers present along the LOS, where the gas quantity is, by construction, unknown. The retrieval itself, which is applied to a narrow $\left(<1 \mathrm{~cm}^{-1}\right.$, see Fig. 3) spectral region surrounding the location of the main methane absorption feature, adopts a two-step approach where contributions by species underlying $\mathrm{CH}_{4}\left(\mathrm{O}_{3}\right.$ in the case of order 182), if any, are retrieved first alone, and then held constant while tentatively retrieving methane in the next step. This separation is done to avoid spurious correlation effects arising whilst trying to jointly infer two species absorbing light at the same wavelengths.

\subsection{Upper limit derivation}

A methane upper limit is considered as a multiple of the $1 \sigma$ uncertainty calculated for the methane LOS density parameter when no convincing $(>5 \sigma)$ detection can be identified in the data. The statistical significance of an $n-\sigma$ definition for an upper limit is challenged in our case by the fact that uncertainty is not dominated by shot noise but by systematic errors which are not normally distributed. This is illustrated by Fig. 3 where spectrum fluctuations in the methane range can be seen to be correlated over more than two consecutive pixels. However, this $1 \sigma$ level is rescaled to account for remaining mismatches in the fitting attempt that the model fails to handle directly. This is generally due to systematic effects that cannot be anticipated and therefore 
corrected during the pre-processing stage and that eventually degrade the quality of the fit. The corresponding rescaling factor (the square-root of the reduced $\chi^{2}$ value) is applied to $\sigma$ only when $\chi^{2}$ is greater than 1 , which therefore forces $\chi^{2}$ to be equal to or less than 1. Although this amounts to truncating the statistics of the fit, it also provides a more conservative estimate of the error bar and thus the upper limit. On average, this rescaling factor is equal to 1.5 , implying that, by the traditional definition, the 1 or $2 \sigma$ reported here correspond to 1.5 or $3 \sigma$ reported in other works.

Lastly, an empirical validation of the upper limit is conducted after every fit by performing an additional fitting attempt whilst artificially injecting the equivalent of a $2 \sigma$ level of methane absorption into the spectrum. An upper limit is considered valid when the retrieved value is close (within $50 \%$ ) to the $2 \sigma$ quantity of injected methane.

\subsection{Theoretical ACS sensitivity}

In order to evaluate the performance of ACS in its detection of methane, a simple exercise was conducted. Applying the retrieval tool described above, we attempted to detect methane using the spectral intervals corresponding to order 182 on a synthetic transmission spectrum containing no gaseous absorption, that is a flat line, and assigned with a variety of $\mathrm{S} / \mathrm{N}$ values per pixel. Through retrieval, we then propagated the error bar assigned to synthetic data points (derived from the specified $\mathrm{S} / \mathrm{N} /$ pixel calculated in the proximity of the $\mathrm{CH}_{4}$ lines of interest) towards the retrieved slant column abundance of methane (in molecules $\mathrm{cm}^{-2}$ ). The result of this theoretical exercise, presented in Extended Data Fig. 3 of K19, gives a log-linear relation between $\mathrm{S} / \mathrm{N}$ and ACS sensitivity to methane, assuming the inferred $1 \sigma$ uncertainty on the retrieved methane represents the ACS sensitivity at a given $\mathrm{S} / \mathrm{N}$. For $\mathrm{S} / \mathrm{N} /$ pixel ranging between 1000 and 10000 , we find the equivalent $\mathrm{CH}_{4} 1 \sigma$ uncertainty to range between $10^{14}$ and $10^{13}$ molecules $\mathrm{cm}^{-2}$. Once ratioed, with an estimate of $\mathrm{CO}_{2}$ slant column density at a certain altitude, the $\mathrm{CH}_{4} 1 \sigma$ uncertainty is then converted into a theoretical upper limit expressed in relative abundance (or VMR). At $12 \mathrm{~km}$ and $25 \mathrm{~km}$, the smallest upper limits - corresponding to an $\mathrm{S} / \mathrm{N}$ of 10000 - that can be theoretically retrieved by ACS are respectively 1 and 10 pptv.

\section{Methane upper limits}

\subsection{Measurement statistics}

An overview of the main characteristics of the entire dataset is given in Fig. 4. The latter consists of 11700 spectra from which retrieval attempts yielded upper limit values clustering in the 10 to 100 pptv range. Of these values, $0.5 \%$ were found below 10 pptv, while $67 \%$ were found in the 10 to 100 pptv range and $32.5 \%$ above 100 pptv (as shown in Fig. 5).

The median value of retrieved $\mathrm{CH}_{4}$ VMR is -0.9 pptv while the median $1 \sigma$ uncertainty is 140 pptv (see Fig. 4A.). Retrievals were only performed below $50 \mathrm{~km}$ as (1) the $\mathrm{CO}_{2}$ signature in order 178 becomes too faint above that altitude and can no longer be used to provide a reliable VMR, and (2) one expects methane to be present in the atmosphere below $50 \mathrm{~km}$ (where more than $99 \%$ of the atmospheric mass is), considering that it has only been measured at the surface or in nadir from orbit.

The retrieved values are associated with a $\chi^{2}$ that is forced not to exceed 1 by adjusting the errors on the measurement vector as previously stated, which, in the case of a dataset
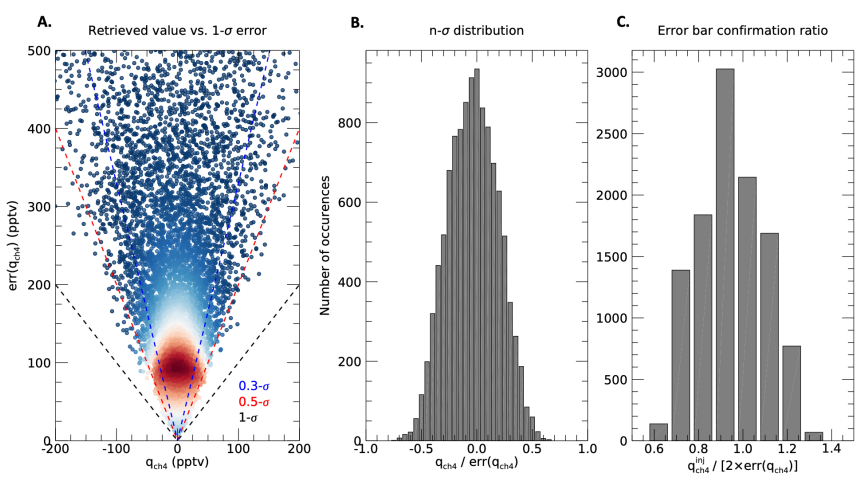

Fig. 4. Panel $A$ : retrieved $\mathrm{CH}_{4}$ VMR values plotted against their corresponding $1 \sigma$ uncertainty, with colours referring to the density of points (redder equals denser), and the horizon corresponding to $0.3,0.5$, and $1 \sigma$ represented by the blue, red, and black dashed lines. Panel B: n $\sigma$ distribution of the retrievals, with n- representing the ratio of the retrieved value over its corresponding $1 \sigma$ error. Panel $C$ : distribution of the upper limit verification index, which is equal to the ratio of the $\mathrm{CH}_{4} \mathrm{LOS}$ density value retrieved after injecting an amount equivalent to the $\mathrm{CH}_{4} 2 \sigma$ error into the spectrum.

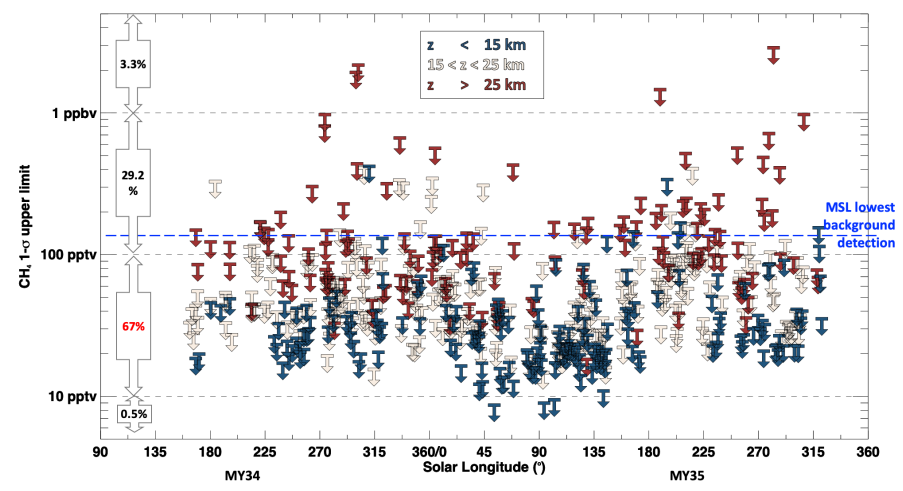

Fig. 5. Altitude distribution of $1 \sigma$ upper limits derived by ACS in three latitudinal regions: $(A)$ mid-to-high southern latitudes, $(B)$ inter-tropical latitudes, and $(C)$ mid-to-high northern latitudes. The blue-to-red colour palette reflects the increasing density of points.

revealing no detection, would induce a normal distribution of the detection $\mathrm{S} / \mathrm{N}$ (in the parameter space), that is, the retrieved value, $\mathrm{q}_{\mathrm{CH}_{4}}$ ratioed by its associated $1 \sigma$ uncertainty, $\operatorname{err}\left(\mathrm{q}_{\mathrm{CH}_{4}}\right)$, bounded by -1 and +1 . This is what we obtain with the ACS dataset: Fig. 4A shows no outlier beyond the horizon corresponding to $\left.\left[\operatorname{abs}\left(\mathrm{q}_{\mathrm{CH}_{4}}\right)\right]=\operatorname{err}\left(\mathrm{q}_{\mathrm{CH}_{4}}\right)\right]$ while Fig. 4B shows the $\mathrm{S} / \mathrm{N}\left(\mathrm{q}_{\mathrm{CH}_{4}} / \mathrm{err}\left(\mathrm{q}_{\mathrm{CH}_{4}}\right)\right.$ is distributed between -0.7 and 0.7 . This ensemble of results therefore indicates no meaningful sign of detection can be identified in the set of 11700 spectra analysed, in concordance with the first report of K19.

The validity of the upper limit dataset can be gauged from Fig. $4 \mathrm{C}$ where the results of the post-retrieval validation results are displayed. Error bar confirmation indices dispersed between 0.7 and 1.3 imply that the algorithm is capable of identifying a methane signature and retrieving the corresponding amount with a $30 \%$ bias or uncertainty in the case where this amount corresponds to twice the estimated upper limit value.

\subsection{Altitude profiles}

Upper limits have a one-to-one correlation with $\mathrm{S} / \mathrm{N}$, itself being governed by the dust attenuation. For this reason, a theoretical altitude (altitude is defined as the distance between the LOS 


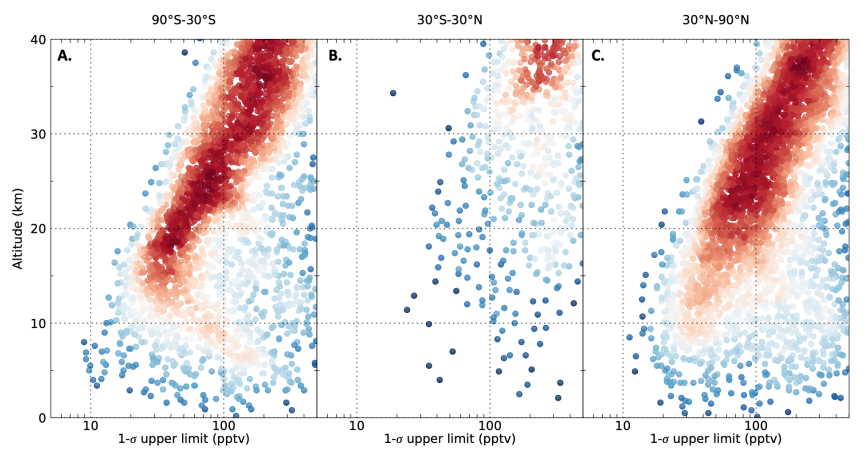

Fig. 6. Temporal evolution of the best $1 \sigma$ upper limits per occultation derived by ACS as a function of solar longitude. Colours represent the three altitude portions (below $15 \mathrm{~km}$, between 15 and $25 \mathrm{~km}$, above $25 \mathrm{~km}$ ) that upper limits correspond to.

tangential point and the surface below it) profile of the LOS density upper limit is shaped primarily by the gradual reduction of dust abundance with altitude (Fig. 6) which subsequently induces higher $\mathrm{S} / \mathrm{N}$ and thus lower upper limits. However, once ratioed with $\mathrm{CO}_{2}$, the vertical trend in retrieved upper limits due to the changing $\mathrm{S} / \mathrm{N}$ is counteracted by the $\mathrm{CO}_{2}$ column density decrease with altitude. Once combined, these two effects end up forcing an optimum altitude for the upper limit determination located between 10 and $30 \mathrm{~km}$ (depending on dust abundance). Below that optimum altitude, the reduction in $\mathrm{S} / \mathrm{N}$ dominates the retrieval, while above it, $\mathrm{CO}_{2}$ column density reduction prevails, generating a 'bended knee' shape in the altitude profile that was expected and presented in Korablev et al. (2018) and also found by Knutsen et al. (2021) with NOMAD data.

Figure 6 is a faithful reflection of this behaviour. The dataset has been recast into three latitudinal regions. The bended knee is only seen outside the equatorial region. Around the equator, ACS sensitivity is hampered by dust and clouds all year long, even during the clear aphelion season, explaining the cluster of values above $35 \mathrm{~km}$. At higher latitudes, profiles reveal the optimum altitude to be located around $15 \mathrm{~km}$ in the southern hemisphere and slightly above $20 \mathrm{~km}$ in the northern hemisphere.

Retaining the best upper limits of every occultation, a filtered view of the dataset is presented in Fig. 7 against altitude and latitude. The trend observed in Fig. 5 is emphasised in this figure with the dispersion of upper limit symbols forming an arch over the equator as a consequence of the ACS performance degradation in that region which is due to dust. In rare cases ( $<10$ occultations), ACS was able to probe below $5 \mathrm{~km}$ altitude, delivering $1 \sigma$ upper limits smaller than $10 \mathrm{pptv}$ ( 20 pptv at $2 \sigma$ ).

\subsection{Seasonal evolution}

Figures 5 and 8 track the seasonal evolution of the derived upper limits, yielding some indication of how these values compare with the background methane detections established by the Curiosity rover (W18). As the dataset encompasses more than a Martian year, it is possible to assess the year-to-year evolution of upper limits, especially during the second half of MY34 when the GDS occurred and which is known to be the dustiest period on Mars and thus the least favourable for ACS detection attempts.

A seasonal signal resulting from the dust seasonal cycle is evident in these plots. As opposed to the previous report of ACS methane upper limits (K19), it was possible this time to probe the aphelion period when dust conditions become more favourable for ACS observations. During that period, the derived ACS upper

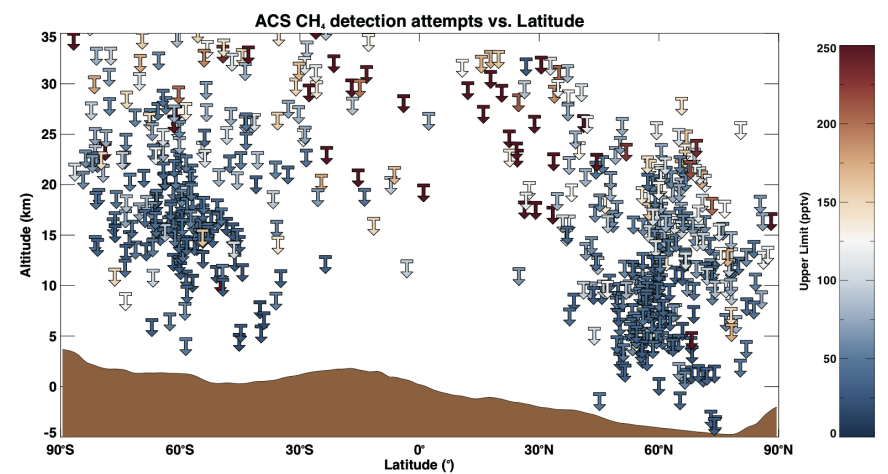

Fig. 7. Altitude distribution of $1 \sigma$ upper limits derived by ACS as a function of latitudes. Upper limit symbols are coloured depending on their value. Mars topography or zonal mean elevation with respect to MOLA zero datum is indicated with the brown shaded area.

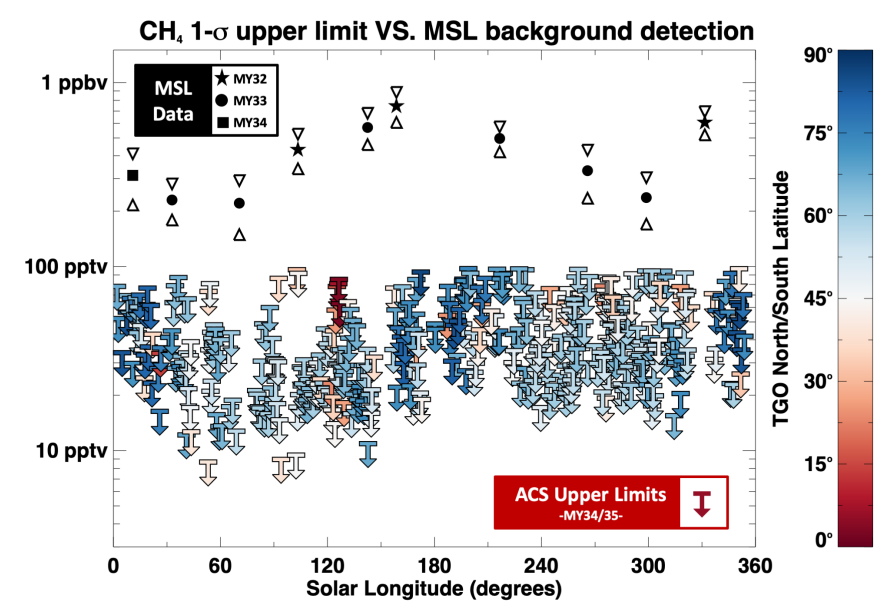

Fig. 8. Annual evolution of the best $1 \sigma$ upper limits (clipped at $100 \mathrm{pptv}$ ) derived at each occultation and plotted as a function of Solar longitude $\left(\mathrm{L}_{\mathrm{s}}\right)$. Upper limit symbols (downward arrows) are coloured depending on their latitude. The values reported by MSL in Webster et al. (2018) concerning the background detections of methane are shown as filled stars (MY32), circles (MY33), and squares (MY34) with their associated error bars.

limits reached their all-time low with several excursions below the 10 pptv level, which were obtained only at the high latitudes $\left(>60^{\circ}\right)$ of both hemispheres. In contrast, the best upper limit of the $30^{\circ} \mathrm{S}-30^{\circ} \mathrm{N}$ region ever recorded lies above $50 \mathrm{pptv}$. Reproducing the same comparison between ACS and MSL as the one shown in K19 (their Fig. 3), Fig. 8 presents an updated version of this latter figure, showing how the two datasets compare with each other over an entire Martian year. Both display a characteristic sine function, the cause of which is different between the two experiments. For ACS, dust directly impacts ACS detection capabilities and thus its capacity to reach the smallest upper limits. For MSL, the origin of the variations is unclear but has been hypothesised to result from a seasonal process (W18), although this hypothesis is not supported by MSL measurement statistics (Gillen et al. 2020). The comparison of the two datasets also reveals a factor of four to five difference between the higher edge of ACS upper limits and the MSL detections.

\subsection{Measurements near Gale crater}

The fact that ACS is impaired by the presence of dust in its attempts to test methane presence in the equatorial region makes comparison with MSL less instructive than it would have been 


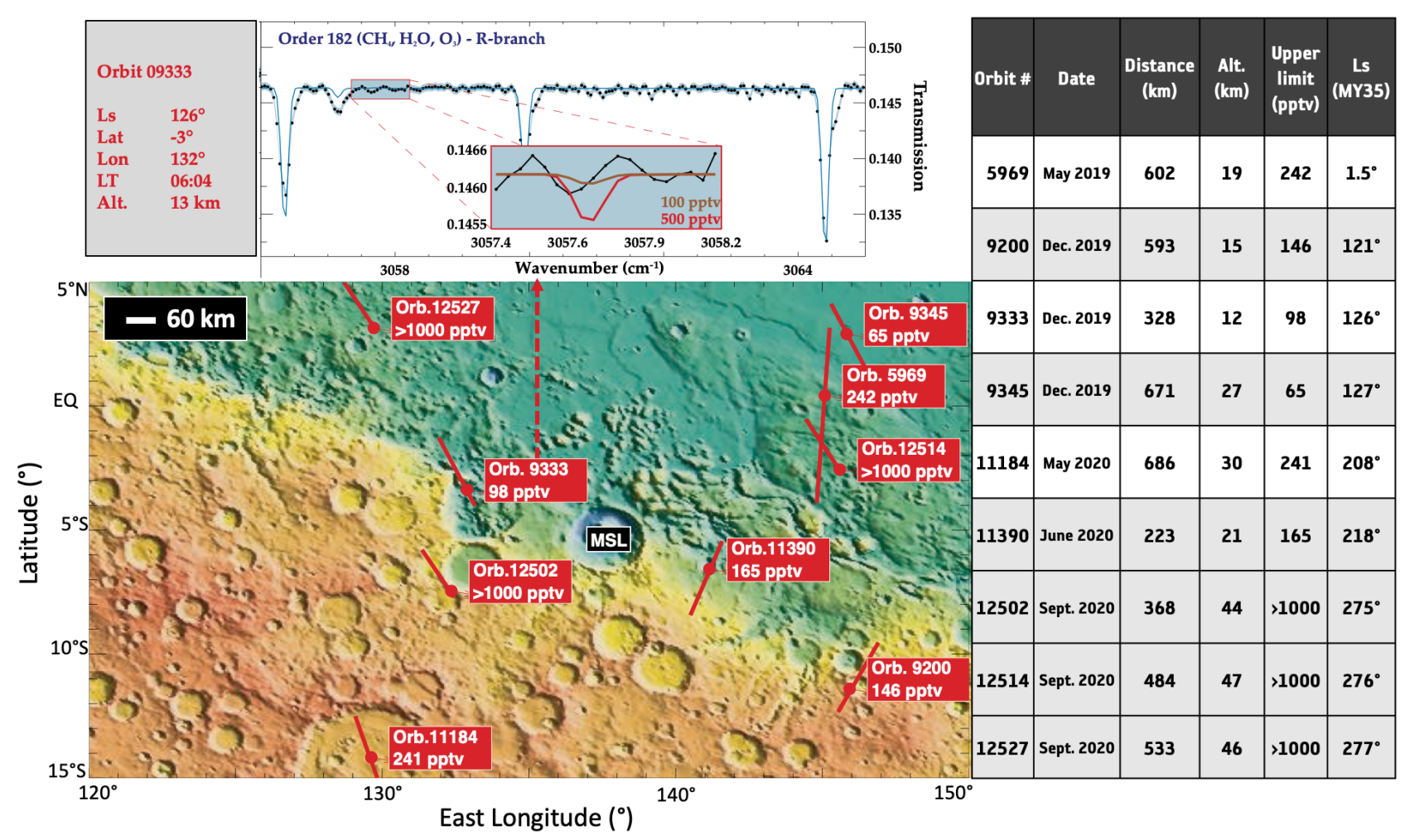

Fig. 9. Locations of all the ACS detection attempts close to Gale crater with their corresponding information given in the table on the right. Red circles correspond to the location where the upper limit was inferred, while the red line extending from it corresponds to the track of the LOS tangent point projected on the surface while its altitude was drifting in the 0 to $50 \mathrm{~km}$ altitude range. The displayed spectrum corresponds to the closest approach that revealed a $\mathrm{CH}_{4}$ upper limit of $\sim 100 \mathrm{pptv}$ and that is compared to a synthetic $\mathrm{CH}_{4}$ signature of 100 and 500 pptv.

elsewhere. However, nine attempts were performed close to the Gale crater within an area with a radius of $\sim 600 \mathrm{~km}\left(10^{6} \mathrm{~km}^{2}\right.$, see Fig. 9). The associated results are reported in the table embedded in Fig. 9, yielding for each attempt the best upper limit and its corresponding geometrical information. Although none of the ACS measurements were performed concurrently with a reported detection by MSL, the fact that a background of methane is suspected to remain all year long in Gale (W18), as reconstructed from measurements spanning three Mars years, is indicative of a preservation or resourcing mechanism that should allow this background to be observed in any given year. Therefore, it can be assumed that all the ACS detection attempts correspond to a time when methane was present in Gale.

The upper limits delivered by ACS range between $\sim 100$ and $450 \mathrm{pptv}$ at altitudes varying from 11 to $45 \mathrm{~km}$. Such altitudes prevent access to the near-surface atmosphere where MSL performs its methane experiments. Nevertheless, there is still justification to conduct a comparison, as the permanent presence of methane in Gale implies a replenishment time of only a few hours, considering that trade winds will evacuate methane from the crater at a speed of 5-8 $\mathrm{m} \mathrm{s}^{-1}$ (Viúdez-Moreiras et al. 2019). The best upper limit produced by ACS near Gale is $100 \mathrm{pptv}$ at $\mathrm{L}_{\mathrm{s}} 126^{\circ}$ on MY34, at a distance of $328 \mathrm{~km}$ from the crater centre, and at an altitude of $11 \mathrm{~km}$. At the same period of $\mathrm{L}_{\mathrm{s}}$, Curiosity measured a background value of 420 pptv at $\mathrm{L}_{\mathrm{s}} 103^{\circ}$ on MY32 and of $650 \mathrm{pptv}$ at $\mathrm{L}_{\mathrm{s}} 159^{\circ}$ on MY33 (W18).

\section{Implications}

This new dataset further confirms that no methane is detected by ACS in the Martian atmosphere. While the previous report by K19 suggested a global absence of methane above a value

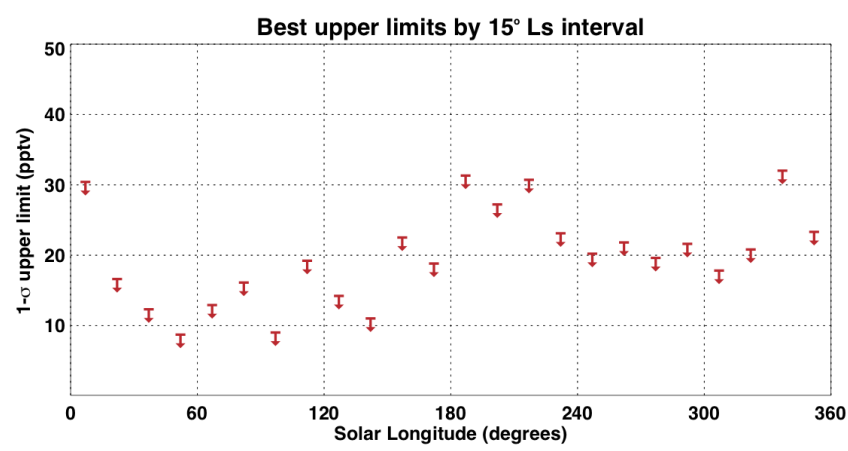

Fig. 10. Seasonal evolution of the minimum upper limits found within $15^{\circ}$ Solar Longitude bins.

of 50 pptv, the present dataset with its sampling of the aphelion season produces even smaller upper limits (see Fig. 10), and is characterised by a value of $\sim 20$ pptv when averaging all the minima of upper limits shown in Fig. 10.

To reconcile the measurements of TGO and those of MSL, Moores et al. (2019) proposed that the methane delivered at night could stagnate at MSL level, permitting its measurement by the TLS experiment, and be dispersed during the day as the planetary boundary layer (PBL) would regain its convective strength and increase in vertical extent. According to this study, such 'breathing' of the PBL could potentially explain why it would then become difficult for TGO to measure methane as it is evacuated from the Gale crater. However, their assumed constraint was that the bulk atmosphere should remain below the 50 pptv upper limit set by TGO. It now seems that a 20 pptv value should be used instead. This new value strongly increases 
the constraints on the unknown fast destruction or sequestration mechanisms needed to create observable variations of methane on Mars (Lefèvre \& Forget 2009; Etiope \& Oehler 2019) and in particular to reconcile the MSL and TGO measurements. Otherwise, assuming the expected methane photochemical lifetime of $\sim 300 \mathrm{yr}$, the source of methane near MSL should be limited in space and/or time to an astoundingly low level, even taking into account the Moores et al. (2019) scenario. Gale should be the only place on Mars where methane is emitted. Considering the $2.7 \times 10^{4} \mathrm{~km}^{2}$ area encompassing Gale crater assumed by Moores et al. (2019) to emit methane in comparison with the $145 \times 10^{6} \mathrm{~km}^{2}$ global surface of Mars, it implies MSL landed in the right spot to measure methane on Mars with a chance of approximately 1 in 5000 to do so.

Assuming Gale is the only place emitting methane and applying the same logic as that employed in K19, which is based on methane filling up the entire Martian atmosphere at a rate of 2 pptv year ${ }^{-1}$, methane outgassed from Gale cannot be older than 10 Mars years.

Mesoscale simulations (Pla-Garcia et al. 2019) suggested that the crater is not strongly isolated at any time of year. The 2019 spike of 19 ppbv recorded by MSL did not occur when ACS was measuring close to Gale. The closest ACS measurement in space and time occurred 4 days later on orbit 7067 (see Fig. 9) at a latitude of $39^{\circ} \mathrm{S}$ and a longitude of $150^{\circ} \mathrm{E}$, that is $\sim 2000 \mathrm{~km}$ away from the centre of Gale crater. At that time, ACS reported an upper limit of $13 \mathrm{pptv}$. If a volume equivalent to $20 \mathrm{ppbv}$ of methane inside Gale was diluted radially in all directions towards the ACS sampled location, then $\sim 20$ pptv could have been measured by ACS, which is not in strong contradiction to its upper limit of $13 \mathrm{pptv}$, considering the assumptions made about methane dilution in this case.

However, specific mesoscale simulations will have to be carried out to better understand how the ACS measurements can potentially help quantify methane lifetime and dispersion out of Gale.

\section{Conclusion}

We processed almost a Mars year and a half of data produced by the ACS instrument in the spectral range encompassing the main mid-infrared absorption band of methane, yielding no methane detection and delivering an annual mean upper limit of $20 \mathrm{pptv}$, thereby refining the first report of K19 that specified an upper limit of $50 \mathrm{pptv}$ for the $\mathrm{L}_{\mathrm{s}} 163-241^{\circ}$ period of MY34. In addition, several measurements performed in the vicinity (less than $700 \mathrm{~km}$ ) of the Gale crater also resulted in no detection. However, out of nine measurements, only three delivered an upper limit - of less than $300 \mathrm{pptv}$ - that can be used to meaningfully constrain the dispersion of methane away from the Gale crater, considering the background values larger than 200 pptv reported by MSL.

This updated dataset further constrains methane lifetime emitted from the Gale crater to about 10 Martian years, a result that relies on the area and the rate of emission assumed by Moores et al. (2019). This lifetime would scale inversely with a smaller or larger emission area or rate, respectively. The seasonal evolution of the retrieved upper limits reveals a strong relation to the dust loading evolution, as anticipated. Because of the specific configuration of the TGO orbit, the intertropical region of Mars remains less densely covered and is also marked by a background of dust supplemented by clouds around aphelion that obscure the incoming solar beam and reduce the performance level of the instrument.
While some improvements in the ACS upper limit determination can be envisioned, the unfavourable seeing conditions above the Gale crater shall remain the strongest challenge for ACS in its attempts to further constrain methane dispersion from where it is putatively emitted. Additional measurements performed during the next aphelion periods, where observing conditions will be optimal, should nonetheless help in identifying the cause of the divergence of results between TGO, MSL, and MEX.

Acknowledgements. The ACS investigation was developed by the Space Research Institute (IKI) in Moscow, and the Laboratoire Atmospheres, Milieux, Observations Spatiales (LATMOS) in Guyancourt. The investigation was funded by Roscosmos, the National Centre for Space Studies of France (CNES) and RSF (Russian Science Foundation 20-42-0903). This work was funded by CNES, the Agence Nationale de la Recherche (ANR, PRCI, CE31 AAPG2019, MCUBE project), the Natural Sciences and Engineering Research Council of Canada (NSERC) (PDF-516895-2018), the UK Space Agency (ST/T002069/1), the UK Science and Technology Facilities Council (ST/R001502/1, ST/P001572/1). All spectral fitting was performed by F.M. The interpretation of the results was done by F.M. and O.K. The preparation of ACS spectra is done at LATMOS by L.B. and at IKI by A.T. Ancillary data are produced in LATMOS by G.L. and in IKI by A.P. Input and aid on spectral fitting were given by K.O. and A.T. The ACS instrument was designed, developed, and operated by A.P., A.S., A.T., F.M., and O.K.

\section{References}

Alday, J., Wilson, C. F., Irwin, P. G. J., et al. 2019, A\&A, 630, A91

Atreya, S. K., Mahaffy, P. R., Niemann, H. B., Wong, M. H., \& Owen, T. C. 2003, Planet. Space Sci., 51, 105

Atreya, S. K., Mahaffy, P. R., \& Wong, A.-S. 2007, Planet. Space Sci., 55, 358

Bertaux, J.-L., Vandaele, A. C., Wilquet, V., et al. 2008, Icarus, 195, 28

Etiope, G., \& Oehler, D. Z. 2019, Planet. Space Sci., 168, 52

Fonti, S., \& Marzo, G. A. 2010, A\&A, 512, A51

Fonti, S., Mancarella, F., Liuzzi, G., et al. 2015, A\&A, 581, A136

Forget, F., Hourdin, F., Fournier, R., et al. 1999, J. Geophys. Res., 104, 24155

Formisano, V., Atreya, S., Encrenaz, T., Ignatiev, N., \& Giuranna, M. 2004, Science, 306, 1758

Geminale, A., Formisano, V., \& Giuranna, M. 2008, Planet. Space Sci., 56, 1194

Gillen, E., Rimmer, P. B., \& Catling, D. C. 2020, Icarus, 336, 113407

Giuranna, M., Viscardy, S., Daerden, F., et al. 2019, Nat. Geosci., 12, 326

Guzewich, S. D., Lemmon, M., Smith, C. L., et al. 2019, Geophys. Res. Lett., 46, 71

Hitchcock, D. R., \& Lovelock, J. E. 1967, Icarus, 7, 149

Knutsen, E. W., Villanueva, G. L., Liuzzi, G., et al. 2021, Icarus, 357, 114266 Korablev, O., Montmessin, F., Trokhimovskiy, A., et al. 2018, Space Sci. Rev., 214, 7

Korablev, O., Avandaele, A. C., Montmessin, F., et al. 2019, Nature, 568, 517 Krasnopolsky, V. A., Maillard, J. P., \& Owen, T. C. 2004, Icarus, 172, 537 Lefèvre, F., \& Forget, F. 2009, Nature, 460, 720

Montabone, L., Spiga, A., Kass, D. M., et al. 2020, J. Geophys. Res., 125, e06111

Moores, J. E., King, P. L., Smith, C. L., et al. 2019, Geophys. Res. Lett., 46, 9430 Mumma, M. J., Villanueva, G. L., Novak, R. E., et al. 2009, Science, 323, 1041 Olsen, K. S., Lefèvre, F., Montmessin, F., et al. 2020, A\&A, 639, A141

Olsen, K. S., Lefevre, F., Montmessin, F., et al. 2021, Nat. Geosci., 14, 67 Perevalov, V. I., Trokhimovskiy, A. Y., Lukashevskaya, A. A., et al. 2021, J. Quant. Spectr. Rad. Transf., 259, 107408

Pla-Garcia, J., Rafkin, S. C. R., Karatekin, Ö., \& Gloesener, E. 2019, J. Geophys. Res., 124, 2141

Sullivan, W. 1969, New York Times, Aug. 8, 1

Trokhimovskiy, A., Perevalov, V., Korablev, O., et al. 2020, A\&A, 639, A142

Villanueva, G. L., Mumma, M. J., Novak, R. E., \& Hewagama, T. 2008, Icarus, 195,34

Viúdez-Moreiras, D., Gómez-Elvira, J., Newman, C. E., et al. 2019, Icarus, 319, 909

Webster, C. R., Mahaffy, P. R., Atreya, S. K., et al. 2013, Science, 342, 355 Webster, C. R., Mahaffy, P. R., Atreya, S. K., et al. 2015, Science, 347, 415 Webster, C. R., Mahaffy, P. R., Atreya, S. K., et al. 2018, Science, 360, 1093 Webster, C. R., Mahaffy, P. R., Atreya, S. K., et al. 2020, A\&A, 641, L3 Zahnle, K., Freedman, R. S., \& Catling, D. C. 2011, Icarus, 212, 493

Zurek, R. W., Chicarro, A., Allen, M. A., et al. 2011, Planet. Space Sci., 59, 284 